\title{
The Use of Near-Infrared Spectroscopy in the Sport-Scientific Context
}

\author{
Kozlová $S^{*}$
}

Sport Research Institute of the Czech Armed Forces (CASRI), Podbabská 3, Czech Republic

*Corresponding author: Kozlová S, Sport Research Institute of the Czech Armed Forces (CASRI), Podbabská 3, Prague 6, 160 00, Czech Republic, Tel: +420721320628, E-mail: kozlova@casri.cz

Citation: Kozlová S (2018) The Use of Near-Infrared Spectroscopy in the Sport-Scientific Context. J Neurol Neurol Disord 4(2): 203. doi: 10.15744/2454-4981.4.203

Received Date: May 4, 2018 Accepted Date: August 7, 2018 Published Date: August 10, 2018

\begin{abstract}
The focus of this research is aimed specifically at the application of near-infrared spectroscopy (NIRS) in exercise and sport science. NIRS is able to monitor the performance of muscle and cerebral tissue and evaluate their role during physical activity. By monitoring tissue oxygenation, an NIRS device is useful in several areas, which may benefit from its applications. In the sport-scientific context, these tools would allow the follow-up of training performance progression over time, the identification of potential limitations, and an evaluation of the effects of therapeutic and rehabilitative interventions. The purpose of the present paper is to assess the current and potential utilization of the NIRS device in exercise and sport science in order to show the potential of this technique. We would like to introduce some trends evoking current interests and opinions in the field. This review article highlights recent studies related to the in vivo assessment of both cerebral and skeletal muscle tissue in the elite sport context using NIRS technology. After a brief description of the basic principles of NIRS measurement and NIRS technology, the work will examine studies carried out in professional athlete populations in a realistic sporting setting, as well as in some areas of application for laboratory use. Based on our findings, we would like to utilize NIRS to objectivize the rate of load and activation of CNS during exercise in professional athletes in future research projects. Among the findings from the presented studies in this review article, NIRS as a method for the functional evaluation of muscle and cerebral tissue oxidative metabolism has been successfully and reliably used in a variety of sporting contexts
\end{abstract}

Keywords: Near-Infrared Spectroscopy; Optical Imaging; Tissue Hemodynamics; Oxidative Metabolism; Muscular Tissue; Cerebral Tissue; Sport Science; Professional Athletes; Elite Sport

List of abbreviations: NIRS: Near-Infrared Spectroscopy; CW NIRS: Continuous-Wave Near-Infrared Spectroscopy; SRS NIRS: Spatially Resolved Spectroscopy; TD NIRS: Time-Domain Near-Infrared Spectroscopy; FD NIRS: Frequency-Domain Near-Infrared Spectroscopy; Hb: Hemoglobin; Mb: Myoglobin; HbO: Oxyhaemoglobin; MbO: Oxymyoglobin; HbR: Deoxyhemoglobin; MbR: Deoxymyoglobin; HbT: Total Tissue Hemoglobin Content; TSI \%: Tissue Oxygen Saturation; FDP: Flexor Digitorum Profundus; O2HTR: Tissue Oxygen Resaturation; SRC: Sport-Related Concussion; TBI: Traumatic Brain Injury; PMS: Premotor Cortex; M1: Primary Motor Cortex; SMA: Supplementary Cortex

\section{Introduction}

Near-infrared spectroscopy (NIRS) is an optical technique that allows the non-invasive continuous measurement of tissue oxygenation and hemodynamic parameters in muscular and cerebral tissue [1-6]. This spectroscopic technique makes use of light at two or more wavelengths in the range of 700-900 nm, which has much better penetration into biological tissue than visible light and to non-invasively monitor and assess not only tissue hemodynamics but also oxidative metabolism [7]. More precisely, NIRS measures changes in the tissue concentrations of oxyhemoglobin ( $\mathrm{HbO}$ )+oxymyoglobin (MbO), deoxyhemoglobin $(\mathrm{HbR})+$ deoxymyoglobin $(\mathrm{MbR})$, total tissue hemoglobin content ( $\mathrm{HbT}=\mathrm{HbO}+\mathrm{HbR})$, and tissue oxygen saturation (TSI \%) [1,4,5] To calculate the changes, an equation of the two- or multiple-wave length method can be applied according to the modified BeerLambert law [6,7].

Typical in vivo NIRS measurements are implemented in reflectance geometry, with the emission and detection fibers positioned on the same tissue's surface [5]. The interaction between near-infrared light and human tissues is dominated by scattering ( $\left.\mu s^{\prime}>>\mu a\right)$ [6]. For muscle measurements, NIRS refers to the skin, the fat layer, the capillary bed, and muscle tissue [5]. In monitoring cerebral tissue, NIR light is emitted through extra-cranial tissue, bone, cerebral-spinal fluid and the surface of the brain through brain tissue and back again where it is collected by a detector [6,8]. NIR light can travel through the tissue where it is diffused and scattered off in random directions and is scattered back up. Source-detector separation needs to be balanced between depth sensitivity and 
signal strength (30 mm is typical in adults) [8]. The pattern the NIR light path follows is a "banana-shaped" curved in which the penetration depth into the tissue approximately corresponds to half of the distance between the light source and the detector $[2,7,8]$. Accordingly, the greater the distance from the detectors, the more light there is, and the greater the depth of measurement is. However, subcutaneous adipose tissue greatly influences NIR signal intensity from the tissue below. Thus, all adult headgear designs now contain at least 1 short separation channel per hemisphere, $15-20 \mathrm{~mm}$, which attenuates the signal by 30-60 \% [6,7].

Measurements of the changes in oxy and de-oxy hemoglobin concentrations are useful for monitoring skeletal muscle and cerebral oxidative metabolism, where the main variable which can be noninvasively evaluated by NIRS is the result of the dynamic balance between $\mathrm{O}_{2}$ utilization and $\mathrm{O}_{2}$ delivery [2]. This can yield relevant information on key physiological and pathophysiological mechanisms, relevant in the evaluation of exercise performance and exercise tolerance in professional athletes [2]. The particular advantage of fNIRS is that it allows recordings during the execution of movement tasks in a natural context. Therefore, NIRS devices have helped to generate the first valuable data in human exercise research [4]. NIRS signals are the result of the weighted average of the $\mathrm{O}_{2}$ saturations of the heme groups of $\mathrm{Hb}$ in the vascular bed (small arteries, arterioles, capillaries, venules, small veins) and of the $\mathrm{Mb}$ heme group in muscle fibers [2]. Detected oxygenation changes mainly reflect changes in the capillary group (Hb-related), from which are coming most of the intravascular NIRS signals and the intracellular $(\mathrm{Mb}-\mathrm{related}) \mathrm{O}_{2}$ levels [2].

NIRS has its role in monitoring the adaptations of individual subjects at all levels of sporting proficiency. For example, elite athletes can use NIRS in their training interventions. It has the ability to detect local, as opposed to global, muscle oxygenation changes in response to training, on the basis of which it can identify the factors limiting exercise tolerance in pre-training, training, post-training and competing environmental conditions [2,3,4,9]. Second, from an exercise physiology point of view, NIRS can potentiality emphasize the reflection of fractional oxygen extraction in an investigated tissue volume for applications on athletes during their rest period or rehabilitation steps $[5,9]$.

The NIRS device is suitable for applications in any population of healthy subjects (both in normal and in altered environmental conditions), patients affected by many diseases, athletes (sports activities), and even infants and children [2,10].

The research areas include investigating brain activation, brain connectivity, functional responses in atypical development (e.g., the British autism study of infant siblings) and many more, such as the sports field which we will describe in greater detail in the following sections $[6,10]$. Using functional near-infrared spectroscopy on structural neuroimaging or an atlas, it is possible to localize which brain areas are being activated [8]. NIRS can be used to identify the brain responding to an activation in response to a specific stimulus and to locate where this activation does or does not occur in the brain $[11,6]$. The area of use involves research applications, such as monitoring the brain oxygenation of newborns in neonatal care and exploring connections between the brain and behavior, e.g. measuring individual differences in action perception and action production $[1,6]$. We can find out if the responses to two types of stimuli are different from one another or if the responses of different subject groups are different from one another, i.e., accelerated recovery of deoxygenation in trained vs. less-trained athletes? Do these responses change over time? [6]. There is so much to explore using this noninvasive technique.

\section{NIRS Technology}

NIRS devices use different technologies. One way to categorize NIRS technologies is by their measurement strategy [6]. The following section will introduce the differences between the variety of approaches to extract information about biological tissues using NIRS systems. In substance, there are four forms of NIRS technology, which are continuous-wave (CW), spatially-resolved spectroscopy (SRS), frequency-domain (FD), and time-domain (or time-resolved) NIRS systems [6,12].

The most widespread approach in commercial and laboratory environments is the continuous-wave (CW) NIRS system, which was originally developed to measure muscle $\mathrm{O}_{2}$ saturation [2]. This modality employs a steady-state light source (e.g., a lamp or LED), which emits constant power over time [5]. Since the introduction of NIRS systems used in monitoring modality, substantial progress has been made using this technology by which the NIRS parameters are recovered [12]. CW NIRS using the parameter recovery technique depends solely on continuously emitted light. This system relies on specific assumptions within an algorithmic reconstruction, a modified Beer-Lambert principle based on a function of wavelength only regarding light scattering through the tissue medium containing chromophores (light-absorbing substances) [12,5]. This modification of the reconstruction algorithm can produce very different results from identical raw NIRS data.

CW NIRS has some critical technical issues, such as the impossibility to extrapolate absolute values for $\mathrm{HbO}$ and $\mathrm{HbR}[12,7,6,5]$. Therefore, CW systems only provide relative quantification, since they just measure intensity. CW systems cannot separate scatter and absorption changes [6]. The other disadvantage is that the signal is influenced by superficial physiological effects and moving artifacts, because the acquired signal is referred to the whole tissue investigated under the probe [5]. For instance, for muscle measurements, it is not straightforward to separate the contributions of these layers. Another critical shortcoming of CW is the assumed different path length, representing the estimated distance travelled by the lights, which limits the accuracy of any interpretation of the output parameters [12]. On the other hand, CW NIRS systems have many advantages. These include: no calibration requirements, inexpensiveness, ease of use, and robustness. CW NIRS can be expanded to numerous channels, or it can be expanded to perform imaging and diffuse optical tomography (DOT) [6]. 
The other NIRS system called spatially-resolved spectroscopy (SRS) allows the pseudo-absolute measurement of cerebral oxygenation that can be compared across individuals and over time [6]. The SRS methodology has been widely adopted in order to improve the validity of CW NIRS. SRS has attempted to enhance the output parameters of multiple CW-NIRS devices, which were manipulated to improve the depth resolution of raw data obtained from CW devices [12]. SRS uses one source and several detectors, and it assumes that intensity decreases linearly with distance. However, the applied assumptions are often invalid [6]. SRS is easy to use and apply, and it is often used in clinical and functional studies.

To extract absolute absorption and scatter, coefficients can be used in frequency-domain (FD) NIRS, which is a progression of the CW concept $[6,12]$. However, cost increases with these qualities. This enhanced technique theoretically allows more consistent quantitative measurements to be derived from biological tissue, particularly in the case of cerebral NIRS where the critical resolution of output parameters from a specific depth is required [12]. FD NIRS measures light intensity attenuation as well as phase shift by using modulated sources $(\sim 100 \mathrm{MHz})[12,6]$. Measuring a phase change allows a determination of the path length travelled by the detected light [6]. The FD device utilizes fiber optic light transfer, as opposed to the CW device which detects light from tissues directly via surface photodiodes [12]. These differences in light detection methods and the highly sensitive phase measurements may contribute to the levels of noise. Noise levels are noticeably greater in the output parameters of an FD device than that seen from a CW device. This effect has been observed in a study comparing output parameters recorded simultaneously from clinically viable NIRO/CW ${ }^{1}$ and ISS/FD ${ }^{2}$ cerebral NIRS devices [12]. The higher level of noise in output parameters produced by an FD device would be a disadvantage within sport-related traumatic injury (TBI) care, where observational conditions could be suboptimal. Davies et al. presumed that NIRS with frequency-domain parameter recovery would demonstrate accurate quantitative measurements and greater consistency than one without this technology, which should be one of the inherent advantages of the FD system [12]. In the study mentioned, both devices demonstrated marked variability in the baseline parameters. Thus, both of them are capable of reliably detecting the change in specific cerebral oxygenation [12]. From the results of this study, FD NIRS does not offer any direct advantage over CW devices in the acute assessment setting, since the FD device did not demonstrate distinguishably different abilities from the CW device in the tested form. However, FD systems are less robust, difficult to expand to imaging, and hard to calibrate [6].

By using time-domain (TD) NIRS, we can extract as much information about photon transport through the tissue as possible. TD NIRS is a different approach using pulsed laser sources. It employs very short pulses of light that are injected into the tissue, and the photon distribution of time of flight (DTOF) is measured after having travelled in the tissue itself $[5,6]$. To time how long it takes light from each pulse to cross the object, extremely sensitive photon-counting detectors are used [6]. From modifications in attenuation and the shape and delay of laser pulses observed in recollected light, it is possible to extrapolate absolute values for tissue optical properties, as well as for $\mathrm{HbO}$ and $\mathrm{HbR}$ concentrations. In addition to that, TD NIRS is capable of separating the contribution to the detected signal coming from different depths, i.e. from the superficial capillary bed, and the one from deeper tissues [5]. A TD-NIRS instrument, which allows the interrogation of slightly deeper portions of tissue, allows an effective mean penetration depth of about $3 \mathrm{~cm}$ and may represent a substantial technical improvement [2]. TD NIRS has the capability to provide absolute measurements of absorption and scattering, and it can even produce 3D whole-volume images [6].

According to studies carried out by utilizing all four types of NIRS instruments, FD and TD techniques are more technologically sophisticated, offer better depth discrimination than that achieved by the CW technique, and allow the absolute characterization of tissue optical properties. From these, it is possible to retrieve the absolute concentration values of [oxy ( $\mathrm{Hb}+\mathrm{Mb})]$ and $[\operatorname{deoxy}(\mathrm{Hb}+\mathrm{Mb})]$ and the derived parameter [total $(\mathrm{Hb}+\mathrm{Mb})]$. However, CW NIRS is less technologically sophisticated, less expensive, but more widely utilized to allow only relative values by just using intensity measurements $[8,16]$.

Davies et al. observed the difference between cerebrally targeted and non-cerebrally targeted NIRS parameters. The probes, specifically targeting brain tissue, observed lower baseline parameters compared to non-neurological somatic tissue in the facial skeleton [12]. The explanation for the difference could be physiological, that is, that the baseline oxygen demand of resting state bone, muscle and skin tissue is significantly lower compared to the brain. However, the specific degree by which superficial tissues influenced the parameters has not been identified. The higher baseline saturation seen in somatic facial tissue indicates that NIRS devices are capable of reliably detecting changes in induced cerebral oxygenation [12]. In terms of intracellular signals detectable by NIRS in the brain cortex, it is possible to assess the redox state of mitochondrial cytochrome oxidase. It can also absorb NIR light, whereas this cannot be done in muscle [2].

The technology of NIRS systems is developing rapidly towards fully wearable devices that can allow measurements in a wide range of environments. Wearable systems have been miniaturized to be able to apply them where other modalities are difficult or impossible to implement, such as in movement studies. With their noninvasive nature, the availability of wearable NIRS systems and the relative insensitivity of NIRS to motion artifacts makes this device an ideal choice for measurements on subjects performing sport exercises. New developments have led many companies to produce portable NIRS devices. For example, a wearable system solution has been developed by NIRx Medical Technologie, LCC (Germany). The NIRSport system combines

${ }^{1}$ The NIRO 200NX (Hamamatsu Photonics, Tokyo, Japan) CW spectrometer measuring at wavelengths of 735, 810 and $580 \mathrm{~nm}$

${ }^{2}$ ISS OxiplexTS (ISS Inc, Champaign IL, USA) FD device measuring at wavelengths of 690 and $830 \mathrm{~nm}$. 
lightweight LED sources and active detectors with innovative strain-relief hardware to create a wearable brain-imaging solution. A wireless CW-NIRS system has been developed by Artinis Medical Systems (The Netherlands), which has a Bluetooth networking module and is about the size of a cell phone. The wireless probes can be attached to the tissue of interest with adhesive tape, and the control modules can either store various amounts of data or send signals to a nearby computer. To date, including those systems mentioned above, only one other wearable device is able to measure both muscle and cerebral tissue. That product is the Lightnirs 8 by Shimadzu (Japan), which incorporates - not LED - but three-wavelength semiconductor lasers and photodiode detectors to provide superior signal quality. It is the most expensive product. To our knowledge, a new wearable NIRS device will be introduced by Gowerlabs (UK) at the fNIRS2018 conference, which will take place in Tokyo, Japan in October 2018. The ability to collect data during human locomotion is why NIRS lends itself to the study of exercise and athletic performance.

\section{Skeletal Muscle Tissue Measurement}

During sport activities, the energy for muscle work substantially comes from oxidative metabolism ${ }^{3}$, which can significantly affect exercise tolerance and performance by its functional limitations or impairments [2]. Applied to athletes, the availability of a reliable tool to noninvasively investigate in vivo, with precision and reproducibility, muscle oxidative metabolism, with a reasonably elevated temporal resolution, therefore appears to be important. Portability, use in outdoor environments, and compatibility with bioelectric measures make fNIRS an optimal choice for any application related to motor execution. Within recent years, the use of NIRS technology to investigate changes in muscle metabolism and muscle oxygenation during physical tasks has rapidly increased $[4,9,13]$. According to the authors, NIRS applied to skeletal muscle can give valuable functional insights into oxidative metabolism and blood flow in muscle in vivo during both exercise as well as in simulated competition in a realistic sporting setting or recovery $[3,9]$. In the following section, we will introduce you to some practical examples of the main topics of NIRS instrument use in the sport-scientific context applied to skeletal muscle. The intention is not to provide complete results with exact figures of the studies mentioned in this review, but to present the current practical potential use of NIRS instruments.

Skeletal muscles represent around $40 \%$ of total body weight, and the majority of them are involved in the total consumption of oxygen VO2 [1]. All regions of the muscle receive nearly fully oxygenated arterial blood. The role of an NIRS instrument is the functional assessment of oxidative metabolism in skeletal muscles during and after exercise using specific measures, such as muscle oxygenation level, deoxygenation rate, and re-oxygenation rate using in vivo applications on human muscular tissue [4]. Individual differences in deoxygenation and re-oxygenation changes can serve as an indication of the training state of the athlete. Neary provided an excellent review of the literature on the use of NIRS in exercise sport science, where he documented the main sports that have used this technology. These include: alpine skiing, cycling, speed skating, treadmill roller skiing, weight lifting, rowing, and running [9]. Recently, there have been several more studies implemented in an applied sports setting, such as shorttrack speed skating, rock climbing, ice hockey, and cycling, with specific measures. These studies monitored changes in oxygen saturation and hemoglobin concentration in muscle microvasculature during the exercises of elite athletes $[3,4]$.

Skeletal muscle fractional $\mathrm{O}_{2}$ extraction determined by NIRS can give us valuable physiological and pathophysiological insights into the integrated responses to exercise, involving different muscle groups, skeletal muscles, and other organs [2]. Geršak et al. were interested in the correlation between muscle activity and its NIRS and EMG signals. Their results show that both NIRS and EMG signals are adequate parameters to determine the beginning of muscle activity increase [1]. Recently, Jones et al. used NIRS to detect improvements in muscle aerobic capacity following training. They demonstrated the potential of utilizing NIRS, as a tool to monitor local muscle oxygen flow and metabolism, and examined the effect of high intensity interval training called SIT (sprint interval cycling) on muscle oxygenation kinetics and performance during an intermittent fitness test (IF) [4]. Twenty-five elite women Olympic hockey players were randomly selected for an experimental training group and control group. The experimental group performed six additional interval trainings over six weeks in addition to their regular training program. In addition, the subjects additionally completed 5 x 30s maximal intensity cycle testing before and after training [4]. Muscle oxygenation of the vastus lateralis muscle of each subject's dominant leg was continuously monitored during sprint cycling using a portable NIRS apparatus [4]. Both the TSI (\%) and HbR + MbR signals presented significant changes in optical measures following SIT, indicative of an increased muscle oxygen extraction capacity [4]. Changes in the group data were seen and reflected in every individual, with each athlete exhibiting increased desaturation of TSI (\%) and increased $\mathrm{HbR}+\mathrm{MbR}$ during post-training sprint efforts [4]. According to the authors, this is the first study showing consistent changes in NIRS parameters and individual performance in all elite athletes following a training program. Based on these findings, NIRS could have the potential for real-time and continuous feedback in the training process, concomitant with training-induced performance improvements with significant alterations in the typical markers of muscle oxidative potential, such as oxygen metabolism [4].

Fryer et al. also explored the relationship between muscle aerobic capacity and the level of sport performance. This study examining the performance of rock climbers aimed to determine whether an index of oxidative capacity in the flexor digitorum profundus (FDP) predicts the best sport climbing red-point grade within the last six months [14]. Recently, it has been suggested that endurance is not primarily dependent on the ability of the brachial artery to deliver oxygen, but rather on the ability of muscle

${ }^{3}$ The oxidation of glucose and lipids in muscle fibers, culminating in oxidative phosphorylation in the mitochondrial respiratory chain. Maximal power by oxidative metabolism and the fraction of this power are intrinsically related to exercise performance and tolerance (Grassi \& Quaresima, 2016). 
to perfuse and use oxygen, i.e., skeletal muscle oxidative capacity [14]. The aim of the study was to examine the independence of forearm flexor endurance in elite climbers to the ability to regulate conduit artery (brachial) blood flow [14]. The oxidative capacity index of the FDP was measured and assessed by NIRS by calculating the half-time for tissue oxygen re-saturation (O2HTR) following 3-5 min of ischemia periods, during which oxygen consumption was measured to evaluate exercise-induced adaptations in muscle mitochondrial function $[5,14]$. The finding of the study suggests that the forearm flexor oxidative capacity index is an important determinant of rock-climbing performance [14].

In a similar vein, Hesford used an NIRS device to monitor right and left leg quadricep oxygenation and blood volume changes to provide an insight into velocity and pacing changes in speed skating [3]. The authors made the measurements of local muscle oxygenation during a simulated competition in short-track speed skating across a range of race distances. Among the results found, there was a significant asymmetry revealed between right and left vastus lateralis muscle oxygen saturation, although at the start of the simulation total muscle hemoglobin concentration decreased equally in both skating legs [3]. When skaters travelled solely on the right leg, there was a decrease in its hemoglobin concentration, whereas an increase was seen in the left leg. This could possibly indicate a difference in muscle hemoglobin concentration during exercise and in resting conditions. In general, during exercise when capillary hematocrit increases, the NIRS signals are higher compared to rest, when the systemic circulation can be very low [2]. This particular study attributes a strong leg asymmetry during the course of subsequent laps and during cornering at high speeds to the specific technical demands placed on the two legs [3].

The use of NIRS to investigate tissue metabolism during acute exercise has exponentially increased within recent years. A measure of reliability was still missing until recently, when Baláš et al. aimed to determine the intersession reliability of NIRS parameters. They addressed questions relating to the reliability of continuous-wave NIRS parameters measuring intermittent handgrip contractions in rock climbers [13]. They aimed to determine the minimal detectable change of CW-NIRS parameters. Exhaustive intermittent handgrip exercise (8-second contraction-2-second relief) were tested on 32 sport climbers at $60 \%$ of maximal voluntary contraction [13]. The authors measured tissue oxygenation in the flexor digitorum profundus by CW NIRS to determine the tissue saturation index (TSI) during each visit. Reliable results for mean deoxygenation during the contractions were provided, and mean muscle re-oxygenation during the relief periods was similarly reliable. This shows CW NIRS to be a reliable measure of deoxygenation and re-oxygenation during intermittent contractions [13].

The published studies show that most authors are using CW-NIRS devices. However, Re, Pirovano, et al. have presented a TDNIRS medical device for muscular oxidative state monitoring for the first time. They used TD NIRS for the measurement of superficial and deep muscle deoxygenation during exercise. Their study considered a series of acquisitions on different muscles that were not yet previously performed with TD NIRS and its custom 3D-printed probe. The probe facilitates its use on moving legs and arms by hosting optical elements for signal acquisition from muscle [5]. The authors employed the device during three different in-vivo measurements in order to assess its capability to acquire a stable and free of movement artifact signal, and to estimate hemodynamic variations. In particular, they examined results during a venous-arterial cuff occlusion of the arm muscle, a cycling exercise, and an isometric contraction of the vastus [5]. The authors suggested a new generation of detectors to improve this medical device. They employed fiber-free SiPM detectors for in vivo TD-fNIRS acquisitions in the study mentioned. These detectors can be put directly in contact with the patient's skin, avoiding the use of optical fibers (which broaden the temporal response of the TD system, reduce collection capability, and attenuate the collected signal).

Following from the above, the application of NIRS can be used to design training programs for athletes by assessing oxidative metabolism during exercise, including the speed of recovery of muscle oxygenation after a training session. It is a viable tool to obtain the necessary information about the supply and utilization of oxygen in specific muscles during dynamic exercise. This is relevant in the evaluation of exercise performance, exercise tolerance, and rehabilitation procedures in professional athletes $[2,3,15]$.

\section{Cerebral Tissue Measurement}

NIRS devices have the ability to detect not only muscle, but also cerebral, activity. Motor execution and fine movements depend on the coordinated action of brain function with peripheral muscles. Though, little is known about what is happening in the brain during physical exercise. Bosch et al. were investigating brain plasticity during physical exercise. They present evidence that 30 min of medium intensity exercise enhances associative memory consolidation [11]. NIRS represents a clinically available and effective brain monitoring modality $[11,12,16]$. It is a viable biomarker to assess the physiological state of the brain. The central nervous system plays a significant role in the control of muscle force production in an extremely flexible manner, both during exercise and in recovery [17]. In this section, we will describe some representative applications of NIRS to human cerebral tissue in order to show the full potential of this technique.

Recent research in brain monitoring has examined changes in the processing of brain activity within the human motor system [16]. In the sport-scientific context, the most common regions of interest of cerebral tissue monitoring are usually the premotor cortex (PMS), the primary motor cortex (M1), and the supplementary cortex (SMA) on both hemispheres [18]. However, it depends on research intention and the resulting area of interest, which may also be prefrontal cortex or primary visual cortex etc. 
[11]. How brain activity within the human motor system changes its processing depending on different barbell load conditions while executing a barbell squat (BS) was the aim of investigation of the recent studies by Kenville [19]. Shibuya et al. examined bilateral M1 activation in response to graded levels of force production during a unilateral finger task [20]. The authors assumed that increased oxygenation reflects cortical activation. Ten right-handed subjects performed a $10 \mathrm{~s}$ finger task at $20 \%, 40 \%$ and $60 \%$ of the maximal voluntary contraction. The authors found symmetrical activation over both M1 areas at all the force levels investigated. These results indicate the ipsilateral M1 takes part in muscle force control.

The mentioned study detected a linear relationship between force and cortical activity in isolated movements, yet it was still unclear whether this relationship applies to compound movements. That was the subject of investigation of the study assessing the feasibility of NIRS for the compound movement barbell squat (BS) and supplementary to the relationship between force levels and cortical activity [19]. During the squat examination, NIRS configurations covered the cortical motor system, the bilateral auditory cortices, the frontopolar areas, and the primary visual cortex, and they incorporated a short-distance channel to use in a short separation regression [19]. The authors assumed that cortical activation would be reflected by increased HbO and a corresponding decline or unchanged $\mathrm{HbR}$. When comparing force levels, the results suggested a relationship between force and cortical activity during the squat, although in the bilateral superior parietal lobule as opposed to the area of the bilateral primary motor cortex as seen in isolated movements [19]. These findings imply a different modulator for compound movements compared to isolated movements. The findings indicate that the previously observed load/force-brain relationship for simple and isolated movements is present in complex, multi-joint movements, such as the BS [19].

The capability of NIRS devices to perform real measurements of tissue oxidative metabolism is of interest for monitoring the training status in athletes as well as their rehabilitation processes [5]. Every year in the U.S., between 1.6 and 3.8 million contactsport participants are affected by sport-related concussions (SRC) [8,21]. This metabolic impairment can cause disproportionate damage to the brain as it is often underestimated. Furthermore, return-to-play criteria based on symptoms may lead to an overestimation in the speed of the recovery. Within the specific context of acute TBI care, any monitoring tool that is readily available, cost effective, and usable by non-specialist stuff should be easily applied and removed with the minimal preparation. NIRS devices meet these criteria [12]. In contrast to neuropsychological and balance tests - which imply impairments that are based on performance, or neuroimaging techniques, where is difficult to realize neural processes - NIRS has the ability to directly measure brain physiology throughout concussion recovery [16,21].

Studies focusing on acute SRC find NIRS as useful systems to evaluate this mild traumatic brain injury (TBI) by detecting the brain hemodynamic impairment of exposed athletes [8,16,21]. Forcione et al. compared cerebral hemodynamic influences using fMRI and NIRS in task-related exercises in acute sport-related concussions. They reported NIRS, in contrast to fMRI, to be an effective tool capable of independently measuring hemodynamic impairment that follows a concussion episode [8]. According to the authors, NIRS should be utilized in the clinical decision-making process regarding brain health. The need for intervention is often required soon after patient contact [12]. For instance, an examination of brain activity during motor tasks is relevant for assessing the return-to-play state of athletes with mild traumatic brain injury (TBI) [21]. The physiological state of the brain following a concussion was an aim of the study by Bishop and Neary, who monitored cerebral oxygenation changes to track concussion recovery using NIRS to assess the relative changes in $\mathrm{HbO}$ and $\mathrm{HbR}$ in the prefrontal cortex [16]. Data were collected from the participants of the study and compared on days 1-4, 4-6, 7-14 of concussion recovery. The data measured resting hemoglobin and hemoglobin changes in response to hypercapnia (five repeated 20s breath holds) [16]. This study shows NIRS to be an objective biomarker that can index severity and the recovery timeline during concussion recovery.

Similar results have been found by Hemlich et al. who used fNIRS to investigate the brain oxygenation of individuals with persistent post-concussion symptoms (pPCS) during postural control [21]. Loss of balance control is one of the pPCS. The authors compared brain oxygenation and postural sway during balance control tasks under six different conditions in three groups. Subjects with pPCS applied more force over time to keep balance in the most difficult balance condition in connection with an increase of cerebral activation, which indicates an increase in attention-demanding processes during postural control [21].

In particular, in the context of neurological pathology such as TBI or pPCS, it is important to be able to disentangle the brain and behavioral correlates. Vergotte et al. provided evidence that the number of networks involved during a motor task significantly correlates with the degree of multifractality found in the sensorimotor outcome [18]. The changes in connectivity in the sensorimotor cortex, which reflect sensory integration, and motor initiation and production were assessed during a prolonged tapping task [18]. The well-known finger-tapping paradigm was used to assess the ability of quick adaptive responses for the purpose of bridging levels of observation to establish a direct relationship between degenerate connectivity patterns enabling adaptation at the brain level and fractal properties as their dynamic signature in the sensorimotor outcome [18]. A breakdown of the fractal properties in pathological compared to healthy subjects supporting the idea that the loss of fractal properties can be considered as a marker of the general loss of adaptability associated with disease.

An intuitive model for studying all brain pathologies involving hypoxic changes provides isocapnic hypoxia [12]. Induced hypoxia, a physiological change pertinent to TBI pathology, was the focus of Davies et al. who examined changes in cerebral tissue saturation during experimental stepwise increases [12]. As observed by the authors, NIRS should be included in clinical practice 
to assess athletes with SRC and to help guide their recovery, safe return to play, and to help avoid a second impact during the window of brain vulnerability [8,21]. Furthermore, hypoxemic hypoxia is a usual part of the physiology during strenuous physical exercise, and it is used to develop athletic performance [12]. Mild, non-damaging intermittent hypoxia is also intentionally used during altitude training adaptation at both the systemic and cellular level [12].

NIRS is a versatile approach to explore the breadth of challenges resulting from this multi-faceted injury, whether it involves rehabilitation or acute evaluation. Following from the above, NIRS measurements serve as an important clue with the potential for the progression of training, as well as the rehabilitation programs of individual athletes. Altogether, the results of these studies show the feasibility of NIRS devices to investigate brain processing in a sport-related context. However, NIRS techniques involve some fundamental challenges due to their sensitivity to superficial hemodynamics. Though NIRS allows measurements in relatively unconstrained environments, movement studies are difficult to implement due to motion artifacts, which occur because of an alteration in optical coupling between the fiber and the scalp [6]. High sensitivity to the movements of NIRS probes and the presence of excessive noise are usually the biggest problems in NIRS measurements [1,6]. Different forms of motion have different effects on NIRS data. The potential postural effects upon the prefrontal hemodynamic response describe an observational study comparing head motion and prefrontal hemodynamics during upright and recumbent cycling exercise. During upright cycling at maximal exercise, head motion was higher, and PETCO2 was lower, despite there being no differences in oxygen uptake or heart rate [15]. In the semi-recumbent posture, prefrontal oxygenation was higher than for recumbent cycling, and deoxygenation and blood volume were higher during upright rather than recumbent and semi-recumbent cycling [15]. This work highlights the robustness of the utility of NIRS devices to track head motion [15]. Nevertheless, the occurrence of motion artifacts can be minimized by the identification of motion artifacts in NIRS data and their correction or removal [6]. Motion artifacts can be minimized by using well-designed, tightly-fitting headgear. Yücel et al. investigated the performance of a new method of reducing motion artifacts, providing improved-optode-scalp coupling through the use of miniaturized optical fiber tips fixed to the scalp using collodion [22,23]. They found that using collodion-fixed fibers reduced the percent signal change of motion artifacts by $90 \%$. Minimizing signal artifacts due to patient movement is especially important in applications where interesting events are linked to subject movement, as is the case in studies set in sport environments.

The studies presented have shown that NIRS represents a brain monitoring modality with many inherent advantages over available alternatives. In terms of clinical application, NIRS devices could be utilized to observe changes in cerebral physiology [11,12].

\section{Conclusion}

The aim of this review was to access the current and potential utilization of NIRS devices in the sport-scientific context. For that purpose, we summarized recent studies related to the measurements of skeletal muscle and cerebral tissue activation by using NIRS technology in a sports context. While some studies have focused on somatic tissue measurements, others have targeted detecting cerebral activity. In view of the above, NIRS as a method for the functional evaluation of muscle and cerebral tissue oxidative metabolism has been successfully and reliably used in a variety of sporting contexts. Based on the research studies presented in this review article, NIRScan offer insights into the physiological and pathophysiological adaptations to conditions of increased $\mathrm{O} 2$ needs involving different organs and systems of the body in an integrated manner [2]. In addition to the intrinsic performance in the presence of movements involving varied postural changes, NIRS is an ideal choice for obtaining measures on subjects performing sport exercises.

In terms of athlete evaluation, NIRS allows tracking the evolution of the rehabilitation process, evaluating the effects of training interventions, and securing training capability and limitation insights [2]. As we know, an improvement in oxidative metabolism inevitably leads to an increase in exercise tolerance, which may represent one of the main determinants of the quality of training program as well as an important predictor of performance improvement. It is postulated that NIRS will provide effective help to improve the efficiency of training modality for elite athletes [2]. Data obtained from the device summarize the effect of training on all the muscle optical parameters and provide valuable information regarding individual elite athletes following a specific training program. Thus, NIRS is useful for monitoring adaptations to training interventions in elite athletes' protocols [4,5]. However, like all neuroimaging methods, NIRS is sensitive to movements, so the presence of motion artifacts is a common problem in NIRS data during movement studies. The applications of NIRS instruments are also limited by depth sensitivity, as well as the number of channels, so it has limited coverage [6]. These challenges can be minimized or even overcome through careful experimental design and data processing methodologies. The other limitation of NIRS is that it can only analyze the superficial layers of the brain [8]. According to some authors, there is another limiting factor, which is the knowledge of spatial priors in all currently available clinical NIRS devices [12]. In future development, clinically viable devices should provide better quantitative measurement of chromophore concentration within cerebral tissue and may help the incorporation of patient-specific atlas(MRI)-based data [12].

Despite the limitations mentioned, some of which have been overcome by recent technological developments, this method has important strengths and presents significant advantages compared to other methods. The applications of NIRS are dictated by their advantages, such as their non-invasive nature and their good temporal and spatial resolution. In addition to their intrinsic performance in the presence of movements, their other benefits include: ease of use, suitability for vulnerable subjects, and good tolerance by infants and vulnerable subjects. And not least, it is also compatible with other modalities, and it is silent [6,12]. 
The major advance has been in its portability. Thanks to all of the above, NIRS can give valuable functional insights into tissue oxidative metabolism in vivo during exercise [2].

Overall, NIRS serves as a promising tool to examine cortical as well as muscle activity in the sport-scientific context. The findings from NIRS measurement serve as an important clue, which have the potential for the translation of measurement findings to performance prediction. NIRS has shown promise as a reliable, non-invasive local measure of oxygen delivery and consumption in elite athletes following training programs. NIRS can serve as an ideal technique for any coach trying to determine the level of fitness of his athletes [1]. Following from the above, with the advent of portable and wearable solutions, NIRS has become the ideal solution for studies set in sport environments intended to evaluate cortical activation within motor actions.

Now, future effort calls for an empirical investigation of muscle and cerebral tissue activation to objectivize the rate of load and activation of CNS during exercise by an NIRS instrument in the professional athlete population and consequently of finding possible ways of increasing the efficiency of training and rehabilitation programs. The potential applications of NIRS to understand cortical brain function during and after exercise in healthy humans should guide our future work. Another possible application which should be further explored in the sport context is regarding muscle fatigue. The link between cortical activity and muscle force generation on fatigue that develops during exercise is not very well understood. Regarding the post-exercise recovery period, little or no data are available concerning the central processes of performance [17]. Future research could focus on recovery processes in the brain following exercise. According to Perrey, NIRS can provide a window on the dynamics of human brain functioning under physiological conditions after exercise. Investigating post-exercise recovery strategies could shed light on the contributions of the brain into the control of muscle force with fatigue development.

\section{References}

1. Geršak V, Geršak G (2009) NIRS: Measuring changes in muscle oxygenation and the detection of muscle activity. In: Proceedings of XIX IMEKO World Congress, Lisbon, Portugal.

2. Grassi B, Quaresima V (2016) Near-infrared spectroscopy and skeletal muscle oxidative function in vivo in health and disease: a review from an exercise physiology perspective. J Biomed Opt 21: 091313.

3. Hesford CM (2012) The use of portable near infared spectroscopy in elite sport. University of Essex, England, UK.

4. Jones B, Hamilton DK, Cooper CE (2015) Muscle oxygen changes following sprint interval cycling training in elite field hockey players. PloS one 10 : e0120338.

5. Re R, Pirovano I, Contini D, Spinelli L, Torricelli A (2018) Time Domain Near Infrared Spectroscopy Device for Monitoring Muscle Oxidative Metabolism: Custom Probe and In Vivo Applications. Sensors 18: 264.

6. Lloyd-Fox S, Pirazzoli L, Blasi A., Cooper R (2018) NIRS Training Course. Birkberck-UCL. Centre for Brain and Cognitive Development, Birkbeck, University of London, Malet Street, London, UK.

7. Hamaoka T, McCully KK, Niwayama M, Chance B (2011) The use of muscle near-infrared spectroscopy in sport, health and medical sciences: recent developments. Philos Trans A Math Phys Eng Sci 369: 4591-604.

8. Forcione M, Colonnese C, Belli A (2018) Cerebral Hemodynamic Influences in Task-Related Functional Magnetic Resonance Imaging and Near-Infrared Spectroscopy in Acute Sport-Related Concussion: A Review. J Imaging 4:59.

9. Neary JP (2004) Application of near infrared spectroscopy to exercise sports science. Can J Appl Physiol 29: 488-503.

10. OxiplexTS (2001) ISS OxiplexTS Brochure (c) 2001 ISS, Inc 1602 Newton Drive, Champaign, Illinois, USA.

11. Bosch BM, Bringard A, Ferretti G, Schwartz S, Iglói K (2017) Effect of cerebral vasomotion during physical exercise on associative memory, a near-infrared spectroscopy study. Neurophotonics 4: 041404.

12. Davies DJ, Clancy M, Lighter D, Balanos GM, Lucas SJE, et al. (2017) Frequency-domain vs continuous-wave near-infrared spectroscopy devices: a comparison of clinically viable monitors in controlled hypoxia. J Clin Monit Comput 31: 967-74.

13. Baláš J, Kodejška J, Krupková D, Hannsmann J, Fryer S (2018) Reliability of near-infrared spectroscopy for measuring intermittent handgrip contractions in sport climbers. J Strength Cond Res 32: 494-501.

14. Fryer S, Stoner L, Stone K, Giles D, Sveen J, et al. (2016) Forearm muscle oxidative capacity index predicts sport rock-climbing performance. Eur J Appl Physiol 116: $1479-84$

15. Tempest GD, Eston RG, Parfitt G (2017) A comparison of head motion and prefrontal haemodynamics during upright and recumbent cycling exercise. Clin Physiol Funct Imaging 37: 723-9.

16. Bishop SA, Neary JP (2017) Assessing prefrontal cortex oxygenation after sport concussion with near-infrared spectroscopy. Clinical physiology and functional imaging 38: 573-85.

17. Perrey S (2015) Investigating the human brain and muscle coupling during whole-body challenging exercise. Front Physiol 6: 285.

18. Vergotte G, Perrey S, Muthuraman M, Janaqi S, Torre K (2018) Concurrent changes of brain functional connectivity and motor variability when adapting to task constraints. Front Physiol 9: 909.

19. Kenville R, Maudrich T, Carius D, Ragert P (2017) Hemodynamic Response Alterations in Sensorimotor Areas as a Function of Barbell Load Levels during Squatting: An fNIRS Study. Front Hum Neurosci 11: 241.

20. Shibuya K, Kuboyama N, Tanaka J (2014) Changes in ipsilateral motor cortex activity during a unilateral isometric finger task are dependent on the muscle contraction force. Physiol Meas 35: 417-28.

21. Helmich I, Berger A, Lausberg H (2016) Neural Control of Posture in Individuals with Persisting Postconcussion Symptoms. Med Sci Sports Exerc 48: 2362-9.

22. Yücel MA, Selb J, Boas DA, Cash SS, Cooper RJ (2014) Reducing motion artifacts for long-term clinical NIRS monitoring using collodion-fixed prism-based optical fibers. Neuroimage 85: 192-201. 
23. Kenville R, Maudrich T, Ragert P (2017) P 16 The implementation of Near-Infrared-Spectroscopy (NIRS) in sports science-a verification of suitability using the compound movement squat. Clin Neurophysiol 128: e334. 